\title{
Can Wearing a Face Mask Protect from COVID-19? A Systematic Review
}

\author{
Ali Mostafaei ${ }^{1,2}$, Sakineh Hajebrahimi ${ }^{1,3,4}$, Fatemeh Sadeghi-Ghyassi ${ }^{1,3}$, Hadi Mostafaei ${ }^{1,5}$, Nasrin \\ Abolhasanpour $^{1,3}$, Amirreza Naseri_, ${ }^{1,3}$, Zahra Sheikhalipour ${ }^{6}$, Hanieh Salehi-pourmehr ${ }^{1,3^{*}}$ (iD
}

1. Research Center for Evidence-based Medicine, Faculty of Medicine, Tabriz University of Medical Sciences, Tabriz, Iran

2. Department of Ophthalmology, Faculty of Medicine, Tabriz University of Medical Sciences, Tabriz, Iran

3. Department of Urology, Faculty of Medicine, Tabriz University of Medical Sciences, Tabriz, Iran

4. Iranian Evidence Based Medicine Center of Excellence and Joanna Briggs Institute (JBI) of Australia, Vienna, Australia

5. Department of Urology, Comprehensive Cancer Center, Medical University of Vienna, Vienna, Austria

6. Department of Medical Surgical Nursing, Tabriz University of Medical Sciences, Tabriz, Iran

ABSTRACT

Background: In the outbreak of infectious diseases, non-pharmacological intervention might be the only available protection tools. The aim of this systematic review is to investigate whether it is or is not necessary to wear masks in new corona virus (COVID-19) outbreaks in the community.

Methods: On February, 28, 2020, related databases were searched with the following keywords: "COVID-19"; "COVID 19"; 2019-nCoV; 2019-CoV; coronavirus; mask* and facemask. We updated the search in March 13, 2020. A total of 982 relevant reports were identified after removing duplicates. Of these, 71 references were screened based on titles and abstracts. After excluding unrelated studies, 36 studies were included in the full-text review and were assessed for eligibility. Finally, 3 articles met our inclusion criteria.

Results: In three wards of hospital with more exposure to infected patients, wearing the N95 respirator while using regular disinfectants and hand hygiene, was a better way to prevent COVID-19 transmission from patients to nurses and physicians when compared to non-users of masks. Another study on family members with a history of travelling to Wuhan, showed that those who had worn a surgical mask only during the hospital visit, were infected. However, the 7 years old child of the family who wore a surgical mask, was not found to be infected by COVID-19. Finally, none of eleven healthcare workers who had unprotected exposure with confirmed cases were infected.

Conclusion: Due to the newness of the COVID-19 virus, no clinical trials have been found regarding the use of the masks in the prevention of the disease, and the level of evidence were low.

Keywords: COVID-19; Mask; Respirator; Systematic Review

Received: 2020/02/22; $\quad$ Accepted: 2020/03/29; Published Online: 2020/04/01

\begin{tabular}{c} 
Corresponding Information: $\begin{array}{l}\text { Hanieh Salehi-pourmehr, Research Center for Evidence-based Medicine, Faculty of Medicine, Tabriz University of Medical } \\
\text { Sciences, Tabriz, Iran. Email: farshiddanesh @ ricest.ac.ir }\end{array}$ \\
\hline (c) (4) 1 Copyright (c) 2020, This is an original open-access article distributed under the terms of the Creative Commons Attribution-noncommercial 4.0 International License which \\
permits copy and redistribution of the material just in noncommercial usages with proper citation.
\end{tabular}

Use your device to scan and read the article online

Mostafaei A, Hajebrahimi S, Sadeghi-ghyassi F, Mostafaei H, Abolhasanpour N, Nasseri A, et al . Can Wearing a Face Mask Protect from COVID-19? A Systematic Review. Iran J Med Microbiol. 2020; 14 (2): 101-107

Download citation: $\underline{B i b T e X}$ | RIS | EndNote | Medlars | ProCite | Reference Manager | RefWorks
Send citation to:
8 Mendeley
Z Zotero
B RefWorks 


\section{Introduction}

On December 31, 2019, Wuhan Municipal in Hubei Province, China, reported a cluster of pneumonia cases with unknown etiology and after one week a novel coronavirus was isolated from the patients; the virus was officially named by the WHO as "COVID-19". The COVID-19 can spread through person-to-person transmission, droplets and aerosols. It was then approved that person-to-person transmission of COVID-19 happens more prominently in hospitals and the disease amplification occurs mostly in health centers $(1,2)$

In the outbreak of infectious diseases, nonpharmacological interventions such as masks and respirators might be the only available protection tools. Since the outbreak of COVID-19, various tools have been used for health care and safety around the world including cotton or gas masks, medical or surgical masks and respirators such as N95, N99, N100, P2, P3, FFP2 and FFP3 which differ based on their design and indication of use (3-5). Some countries like Singapore, did not recommend healthy people to use masks but in neighboring Malaysia and Thailand, the government urged people to always have masks ready. Conflicting messages have showed confusion over how to protect against COVID-19 (6).

One of the useful experiences learned from Severe Acute Respiratory Syndrome (SARS) contagion was detecting the protective role of wearing masks against SARS and other viral infections. WHO considers using hand hygiene and masks as preventive measures to limit the spread of COVID-19 in infected areas (7). Therefore, this systematic review aims to investigate whether it is or is not necessary to wear masks in coronavirus outbreaks in the community.

\section{Materials and Methods}

This systematic review and meta-analysis is conducted based on the Cochrane Collaboration Handbook and PRISMA Statement (Preferred Reporting Items for Systematic Reviews and Meta-Analyses) (14). Databases including PubMed, Embase, Medline (via Ovid), Cochrane library, Up-To-Date, WHO, TRIP database and Google Scholar were searched on February 28, 2020, to find possible related evidence. For this purpose, the following keywords were searched: "COVID-19"; "COVID 19"; 2019-nCoV; 2019-CoV; coronavirus; mask* and facemask. We updated the search in March 13, 2020.

The search strategy was set to each database and proper keywords were searched when possible. A total of 982 relevant reports were identified after removing duplicates. Of these, 71 references were screened based on titles and abstracts. After excluding unrelated studies, 36 studies were included in the full-text review and were assessed for eligibility. Finally, a retrospective study and a case series study met our inclusion criteria for data extraction. Also we updated the search at March 13, 2020 for any more relevant papers. In this stage we found 140 more studies, in which after screening, only one study met our inclusion criteria and was added to our result (Figure 1)

\section{Results}

\section{Mask Protection from COVID-19 in Healthcare Setting and Community}

Wang et al. (8) retrospectively collected infection data in the time interval between January 2 to January 22, 2020, in six different departments (Lung, ICU, Infection, Pancreatic Hepatobiliary Surgery, Trauma, Microsurgery, and Urology) from Zhongnan Hospital of Wuhan University. Health care providers of respiratory, ICU and infectious diseases department who used N95 respirators, disinfectants and frequently hand cleansers, were enrolled in the study as "the N95 group". Given the lack of awareness of COVID-19 in the early days of the outbreak, medical staff in the other three wards of the hospital did not use any medical masks and occasionally used disinfectants and hand hygiene. This group was considered "the no-mask group". The cases suspected to COVID-19 infection were diagnosed with chest CT and were confirmed by molecular diagnostic methods. Of the total patients, 28 confirmed and 58 suspected cases were diagnosed during the study data collection period. Medical staff contact with COVID-19 patients in the N95 group was significantly greater than in the no-mask group (for patients confirmed: difference: 733; odds ratio: 8.33$)$.

The results of Wang et al. showed that of 493 medical staff, there were no cases of infection among nurses (222) or physicians (56) in the N95 group. In the no-mask group, the infection was observed in 10 medical staff (out of a total of 77 physicians and 136 nurses) (8). Regardless of the high level of exposure to the COVID-19 virus in the N95 group, the infection rate in medical staff in the group using this mask was lower than that of the group that did not use the mask. (Difference: 4.65\%, (95\% Cl: 1.75\%infinite)) (Adjusted odds ratio (OR): 464.82, (95\% Cl: 97.73-infinite)). This study was not peer-reviewed and therefore the level of evidence was low.

In another study carried out by Chan et al., (9) epidemiological, clinical, laboratory, radiological and microbiological findings as well as phylogenetic properties were analyzed in six family members (four adults and two children) with symptoms of unexplained pneumonia who had travelled from Shenzhen, Guangdong Province to Wuhan, as well as another family member who did not travel to Wuhan (7 family members in total). 


\section{PRISMA 2009 Flow Diagram}
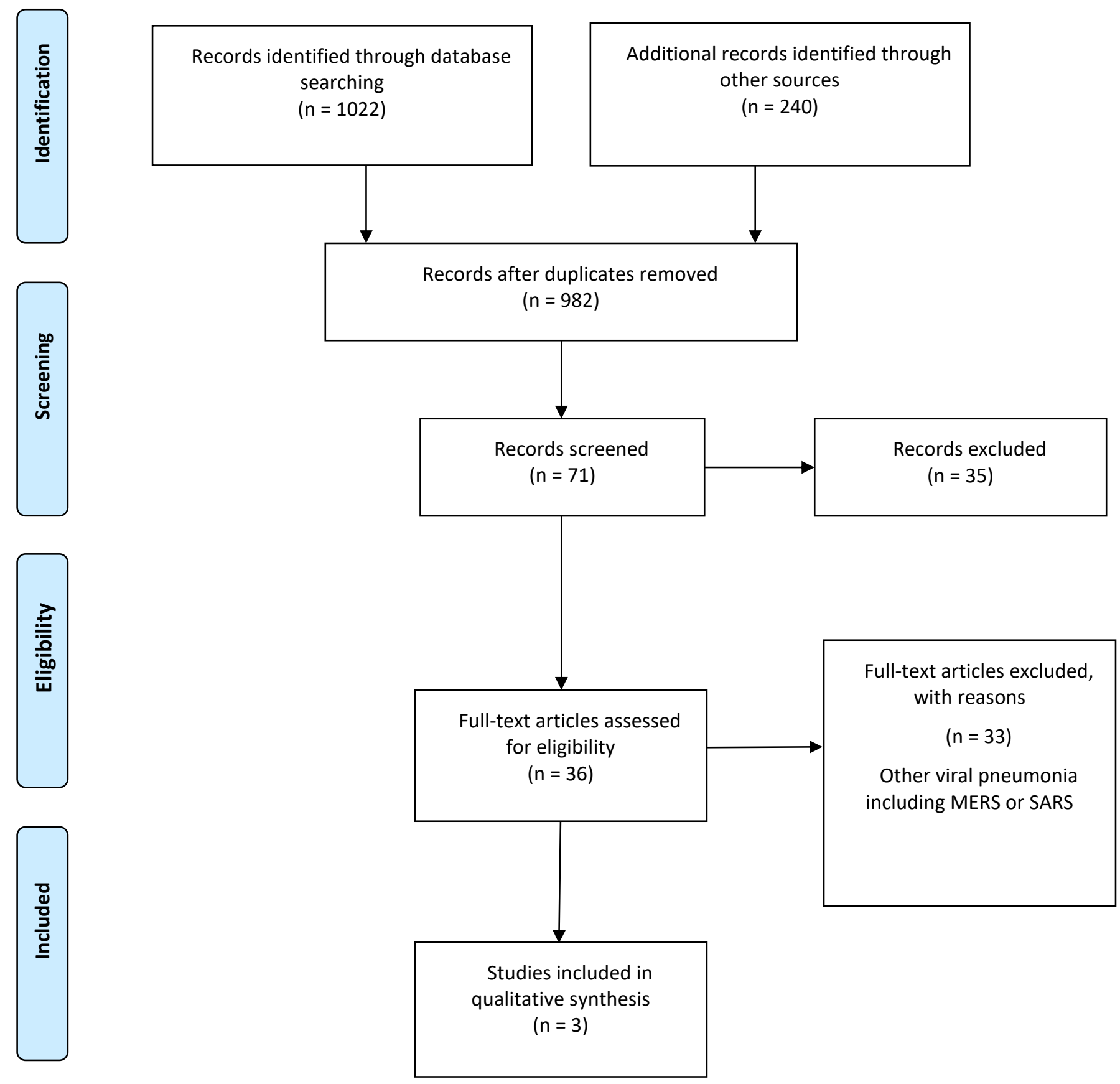

Figure 1. Flowchart of searching and selecting the final studies 
Of the six family members who traveled to Wuhan, five (four adults and one 10-year-old child) were infected with the new coronavirus. In addition, the family member who had not traveled to Wuhan was infected with the virus after several days of contact with the infected family members. None of the family members had a visit or any other contact with markets or animals; however, two members of this family had visited the Wuhan hospital. Three to 6 days after exposure in the five family members, signs of fever, upper or lower respiratory tract symptoms, diarrhea and a combination of these symptoms were observed (9). The radiological ground-glass lung opacities were observed in them and one asymptomatic child (aged 10 years), too. One of the patients had worn a surgical mask during the hospital visit. However, the 7 year old child who wore a surgical mask for most of the time in Wuhan, was not found to be infected by COVID-19.

A study in Hong Kong society in the first 42 days after announcement of a cluster of pneumonia in China, could maintain zero nosocomial transmission of COVID-19 since the importation of first confirmed case since day 22. Eleven of 413 healthcare workers caring patients before confirmation of COVID-19, had unprotected exposure with confirmed cases and none of them was infected. Also, they couldn't detect RNA of virus in 8 air samples collected at a distance of $10 \mathrm{~cm}$ of patient's chin. This suggested COVID-19 is not transmitted by airborne route, whether the conclusion based on analysis of one patient is not reliable. It can also be because of rapid dilution of air inside airborne infection isolation room, or airborne route, too. Anyway, nosocomial transmission of COVID-19 was not observed and according to the results of this study, wearing surgical mask alone was not associated with protection of person from acquisition of COVID-19 (10).

\section{Mask Protection in the Other Corona Viral Infections in Community Settings}

Due to the newness of the COVID-19 virus, no clinical trial was found on the efficacy of different types of masks in preventing the disease among health workers or in community settings. However, several randomized clinical trials (RCTs) have been performed using the mask in line with extensive interventions (such as mask and handwashing) in the community and therapeutic centers and showed various outcomes in preventing transmission in occasional outbreaks of infectious diseases, like influenza.

Among the nine clinical trials that used the mask in the community settings, all studies except for one used the mask for respiratory protection. They found that using the mask alone and mask in addition to hand hygiene may be beneficial for protection from infection in community settings, indeed, if used early and adhered (11-19). However, the total level of evidence available to determine the effectiveness and efficiency of the mask alone is low to moderate due to the use of complex interventions.

\section{Mask Protection in the Other Corona Viral Infections in Health Care Settings}

We found four RCTs reporting the clinical efficacy of using masks or respirators among healthcare staff (2023). The first RCT was conducted on 32 health workers in Japan. Health care providers were divided into two groups; a group using the medical mask and a control group. There was no significant difference in the occurrence of respiratory diseases between the two groups $(P=0.81)(20)$.

Another study in Canada compared the use of medical masks with N95 respirators on 44 nurses and showed a nearly equal effect for both masks in preventing influenza (23.6\% in medical mask vs. 22.9\% in N95 respirators users; absolute risk difference: $-0.73 \%, 95 \%$ confidence interval $-8.8 \%$ to $7.3 \%$ ). However, due to the lack of a control group in this study, the effectiveness of the masks cannot be evaluated (due to the high incidence of influenza in both groups) (21). Another study also reported a similar rate $(23 \%)$ of influenza among health care providers who did not use the mask (24).

Table 1. Summery of studies characteristics included in systematic review.

\begin{tabular}{|c|c|c|c|c|}
\hline Reference & Population & Mask type & Infected with mask & Infected without mask \\
\hline $\begin{array}{c}\text { Wang et al. } \\
\text { (8) }\end{array}$ & 706 medical staff & N95 & None of 493 & 10 of 213 \\
\hline $\begin{array}{c}\text { Chan et al., } \\
\text { (13) }\end{array}$ & 6 family members & surgical mask & None of 1 & 5 of 5 \\
\hline $\begin{array}{c}\text { Cheng et al., } \\
\text { (14) }\end{array}$ & $\begin{array}{l}11 \text { healthcare } \\
\text { workers }\end{array}$ & Surgical mask & None of 402 & None of 11 \\
\hline
\end{tabular}


The third trial conducted on 1922 Chinese healthcare workers, compared the outcome in three groups including the use of the medical mask, use of the N95 respirator and a control group. $\mathrm{N} 95$ respirator had a protective role against clinical respiratory infections (odds ratio: 0.38, 0.17 to 0.86); however, it did not resist PCR-confirmed influenza. The overall results showed that since the highest infection rate was in the control group, the $\mathrm{N} 95$ respirator users observed a low infection rate . (22)

The fourth RCT was performed on 1669 health care workers in China to investigate the consequences of continued use of the $\mathrm{N} 95$ respirators and medical masks during high-risk procedures. The results of this study showed that the N95 respirator is very useful against clinical respiratory infections (hazard ratio: $0.39,0.21$ to 0.71 ) and bacterial colonization (hazard ratio: $0.40,0.21$ to 0.73$)(23)$

\section{Discussion}

Any severe acute respiratory infections (SARI) among emergency medical staff require urgent examinations. Early diagnosis of SARI symptoms with unknown causes in medical treatment centers through a rapid triage and isolation system for suspected or confirmed COVID-19 infections has been strongly recommended to prevent and control the infection (25-27). Medical and cloth masks have been designed to prevent the person wearing the mask from spreading the infection and to protect the person from being contaminated through the blood or body fluids of patients. Furthermore, the respirator is a useful option to protect the person against respiratory infection (3-5).

The results of the present study showed that N95 respirator, with respect to hand hygiene and the use of disinfectants can reduce the incidence of COVID-19 in health care providers and in the other study, the use of medical mask decreased the rate of infection in one 7 years old child. A model study suggested that using the mask in the community may delay the pandemic outbreak of infection, although efficacy estimates were not based on clinical trial (28).

For people with no respiratory symptoms, medical masks are not required in the community, even if COVID19 was an outbreak in the area. However, wearing masks is recommended in crowded environments (such as public transport) and for the high-risk individuals (elderly, pregnant women and people with underlying diseases). The use of masks does not diminish the importance of other public measures to prevent infections and may lead to unnecessary costs and provisions (29).

Lee et al. demonstrated that due to the unknown nature of the COVID-19 virus and insufficient knowledge of its transmission, hand washing and hygiene together with the use of eye protection and masks are protective recommendations for all health care providers (30). However, the extra demand for preparing a mask sometimes is a serious concern among the general public. Many people go to the pharmacy to purchase masks, which leads to decreased medical facilities in the emergency care centers (31).

In previous studies on investigation of Middle East Respiratory Syndrome-Coronavirus (MERS-CoV) Infection, it was recommended that wearing medical mask is necessary only in close contact ( 1 meter) and when health workers enter the room of patients (32). However, $\mathrm{Xu}$ et al. demonstrated that the most important material of novel coronavirus is the RNA that controls its replication and metabolism. The RNA strands are light, thus can freely float in the air and cause infection through contamination. Therefore, filtering gas mask can be beneficial tools to remove the novel coronavirus RNA strands that are connected to droplets and dust, but not for filtering the free RNAs in the air. They recommended isolated gas masks to hospital personnel that are in direct contact with patients (33). A study of immediate psychological responses during the Initial stage of COVID-19, found that wearing respirators, was associated with lower levels of depression and anxiety in society. Although the WHO emphasizes that masks are effective only when used in combination of hand hygiene, wearing a mask could offer potential psychological benefits by offering a sense of security (34). Despite the reported psychological benefits, using mask can be harmful too; because the low level of anxiety can lower society attention to healthcare system recommendations like hand hygiene and quarantine.

\section{Conclusion}

The results of the present study showed that respirator N95, with respect to hand hygiene and use of disinfectants can reduce the incidence of COVID-19 in health care providers and in another study, the use of medical mask reduced the rate of infection. Due to the newness of the COVID-19 virus, no clinical trials have been found regarding the use of the mask in the prevention of the disease. However, using masks in different community settings can protect if used early and on time. For health care providers, evidence suggests that respirators are more beneficial than masks during the epidemics and outbreaks. Respirators are also recommended if the disease is severe and when mortality is high and no medication or vaccine is available.

Strategy and guidelines for mask use in the entire world are inconsistent; possibly reflecting the relatively low number of RCTs available. Ultimately, the biggest priority is to provide evidence-based options to health care providers who need to be confident about their 
health and occupational safety and to ensure the integrity of an effective response to the epidemic.

\section{Key Points}

- In the outbreak of infectious diseases, non-pharmacological interventions such as masks and respirators might be the only available protection tools.

- $\quad$ N95 respirator, with respect to hand hygiene and the use of disinfectants, can reduce the incidence of COVID-19 in health care providers.

- World Health Organization recommended that for people with no respiratory symptoms, medical masks are not required in the community.

- Wearing masks is recommended in crowded environments (such as public transport) and for the high-risk individuals (elderly, pregnant women and people with underlying diseases).

\section{Acknowledgment}

Noun

\section{Conflict of Interest}

Authors declared no conflict of interests.

\section{Rerefencess}

1. Chen, N., et al., Epidemiological and clinical characteristics of 99 cases of 2019 novel coronavirus pneumonia in Wuhan, China: a descriptive study. The Lancet, 2020. 395(10223): p. 507-13. [DOI:10.1016/S0140-6736(20)30211-7]

2. Organization, W.H., Clinical management of severe acute respiratory infection when novel coronavirus ( 2019-nCoV) infection is suspected: interim guidance, 28 January 2020. 2020, World Health Organization.

3. Liverman $\mathrm{CT}$, Larson $\mathrm{EL}$, editors. Preventing Transmission of Pandemic Influenza and Other Viral Respiratory Diseases: Personal Protective Equipment for Healthcare Personnel: Update 2010. National Academies Press; 2011 May 26.

4. Siegel, J.D., et al., 2007 Guideline for isolation precautions preventing transmission of infectious agents in healthcare settings. 2007. [DOI:10.1016/j.ajic.2007.10.007] [PMID] [PMCID]

5. Maclntyre, C.R. and A.A. Chughtai, Facemasks for the prevention of infection in healthcare and community settings. Bmj, 2015. 350: p. h694. [DOI:10.1136/bmj.h694] [PMID]

6. Geddie, J.S.J., To Mask or Not to Mask: Confusion Spreads over Coronavirus Protection. Available online: https://uk.reuters.com/article/us-chinahealth-masks-safety/to-mask-or-not-to-maskconfusion-spreads-over-coronavirus-protectionidUKKBN1ZUOPH, 2020.
7. Ung, C.O.L., Community pharmacist in public health emergencies: Quick to action against the coronavirus 2019-nCoV outbreak. Research in Social and Administrative Pharmacy, 2020. [DOI:10.1016/i.sapharm.2020.02.003] [PMID]

8. Wang, X., Z. Pan, and Z. Cheng, Association between 2019-nCoV transmission and N95 respirator use. medRxiv, 2020. [DOI:10.1016/j.jhin.2020.02.021]

9. Chan, J.F.-W., et al., A familial cluster of pneumonia associated with the 2019 novel coronavirus indicating person-to-person transmission: a study of a family cluster. The Lancet, 2020. 395(10223): p. 514-23. [DOI:10.1016/S0140-6736(20)30154-9]

10. Cheng, V.C., et al., Escalating infection control response to the rapidly evolving epidemiology of the Coronavirus disease 2019 (COVID-19) due to SARSCoV-2 in Hong Kong. Infection Control \& Hospital Epidemiology, 2020: p. 1-24. [DOI:10.1017/ice.2020.58]

11. Cowling, B.J., et al., Preliminary findings of a randomized trial of non-pharmaceutical interventions to prevent influenza transmission in households. PloS one, 2008. 3(5). [DOI:10.1371/journal.pone.0002101] [PMID] [PMCID]

12. Cowling, B.J., et al., Facemasks and hand hygiene to prevent influenza transmission in households: a cluster randomized trial. Annals of internal medicine, 2009. 151(7): p. 437-46. [DOI:10.7326/0003-4819151-7-200910060-00142] [PMID]

13. Maclntyre, C.R., et al., Face mask use and control of respiratory virus transmission in households. Emerging infectious diseases, 2009. 15(2): p. 233. [DOI:10.3201/eid1502.081166] [PMID]

14. Aiello, A.E., et al., Mask use, hand hygiene, and seasonal influenza-like illness among young adults: a randomized intervention trial. The Journal of infectious diseases, 2010. 201(4): p. 491-498. [DOI:10.1086/650396] [PMID]

15. Larson, E.L., et al., Impact of non-pharmaceutical interventions on URIs and influenza in crowded, urban households. Public Health Reports, 2010. 125(2): $\quad$ p. $178-191$. [DOI:10.1177/003335491012500206] [PMID] [PMCID]

16. Canini, L., et al., Surgical mask to prevent influenza transmission in households: a cluster randomized trial. Plos one, 2010.2 5(11). [DOI:10.1371/journal.pone.0013998] [PMID] [PMCID]

17. Simmerman, J.M., et al., Findings from a household randomized controlled trial of hand washing and face 
masks to reduce influenza transmission in Bangkok, Thailand. Influenza and other respiratory viruses, 2011. 5(4): p. 256-67. [DOI:10.1111/i.17502659.2011.00205.x] [PMID] [PMCID]

18. Aiello, A.E., et al., Facemasks, hand hygiene, and influenza among young adults: a randomized intervention trial. PloS one, 2012. 7(1). [DOI:10.1371/journal.pone.0029744] [PMID] [PMCID]

19. Suess, T., et al., The role of facemasks and hand hygiene in the prevention of influenza transmission in households: results from a cluster randomised trial; Berlin, Germany, 2009-2011. BMC infectious diseases, 2012. 12(1): p. 26. [DOl:10.1186/14712334-12-26] [PMID] [PMCID]

20. Jacobs, J.L., et al., Use of surgical face masks to reduce the incidence of the common cold among health care workers in Japan: a randomized controlled trial. American journal of infection control, 2009. 37(5): p. 417-19. [DOI:10.1016/j.ajic.2008.11.002] [PMID]

21. Loeb, M., et al., Surgical mask vs N95 respirator for preventing influenza among health care workers: a randomized trial. Jama, 2009. 302(17): p. 1865-71. [DOI:10.1001/jama.2009.1466] [PMID]

22. Maclntyre, C.R., et al., A cluster randomized clinical trial comparing fit-tested and non-fit-tested N95 respirators to medical masks to prevent respiratory virus infection in health care workers. Influenza and other respiratory viruses, 2011. 5(3): p. 170-9. [DOI:10.1111/j.1750-2659.2011.00198.x] [PMID] [PMCID]

23. Maclntyre, C.R., et al., A randomized clinical trial of three options for $\mathrm{N} 95$ respirators and medical masks in health workers. American journal of respiratory and critical care medicine, 2013. 187(9): p. 960-6. [DOI:10.1164/rccm.201207-11640C] [PMID]

24. Elder, A.G., et al., Incidence and recall of influenza in a cohort of Glasgow healthcare workers during the 1993-4 epidemic: results of serum testing and questionnaire. Bmj, 1996. 313(7067): p. 1241-2. [DOI:10.1136/bmj.313.7067.1241] [PMID] [PMCID]

25. Reich, D.S., C.F. Lucchinetti, and P.A. Calabresi, Multiple Sclerosis. The New England journal of medicine, 2018. 378(2): p. 169-80. [DOI:10.1056/NEJMra1401483] [PMID] [PMCID]
26. Kingwell, E., et al., Incidence and prevalence of multiple sclerosis in Europe: a systematic review. BMC neurology, 2013. 13: p. 128. [DOI:10.1186/1471-2377-13-128] [PMID] [PMCID]

27. Post, M.W., Definitions of quality of life: what has happened and how to move on. Top Spinal Cord Inj Rehabil, 2014. 20(3): p. 167-80. [DOI:10.1310/sci2003-167] [PMID] [PMCID]

28. Brienen, N.C., et al., The effect of mask use on the spread of influenza during a pandemic. Risk Analysis: An International Journal, 2010. 30(8): p. 1210-18. [DOI:10.1111/j.1539-6924.2010.01428.x] [PMID]

29. Feigin VL, Abajobir AA, Abate KH, Abd-Allah F, Abdulle AM, Abera SF, Abyu GY, Ahmed MB, Aichour AN, Aichour I, Aichour MT. Global, regional, and national burden of neurological disorders during 1990-2015: a systematic analysis for the Global Burden of Disease Study 2015. The Lancet Neurology. 2017 Nov 1;16(11):877-97.

30. Li, J.-P.O., et al., Novel Coronavirus disease 2019 (COVID-19): The importance of recognising possible early ocular manifestation and using protective eyewear. 2020, BMJ Publishing Group Ltd. [DOI:10.1136/bjophthalmol-2020-315994] [PMID]

31. Hui, D.S., et al., Exhaled air dispersion distances during noninvasive ventilation via different Respironics face masks. Chest, 2009. 136(4): p. 9981005. [DOI:10.1378/chest.09-0434] [PMID] [PMCID]

32. Ajlan, A.M., et al., Middle East respiratory syndrome coronavirus (MERS-CoV) infection: chest CT findings. American Journal of Roentgenology, 2014. 203(4): p. 782-7. [DOI:10.2214/AJR.14.13021] [PMID]

33. $\mathrm{Xu}, \mathrm{Z}$. and S. Asakawa, Can the novel coronavirus transmit via RNAs without protein capsids? 2020. [DOI:10.31219/osf.io/4nj6e]

34. Wang, C., et al., Immediate Psychological Responses and Associated Factors during the Initial Stage of the 2019 Coronavirus Disease (COVID-19) Epidemic among the General Population in China. International Journal of Environmental Research and Public Health, 2020. 17(5): p. 1729. [DOl:10.3390/ijerph17051729] [PMID] [PMCID] 